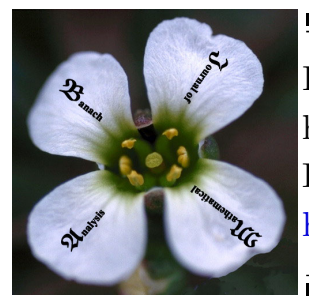

Banach J. Math. Anal. 9 (2015), no. 2, 183-195

http://doi.org/10.15352/bjma/09-2-13

ISSN: $1735-8787$ (electronic)

http://projecteuclid.org/bjma

\title{
SOME HOMOLOGICAL AND COHOMOLOGICAL NOTIONS ON T-LAU PRODUCT OF BANACH ALGEBRAS
}

\author{
MEHDI NEMATI ${ }^{1}$ AND HOSSEIN JAVANSHIRI*2 \\ Communicated by Y. Zhang
}

\begin{abstract}
Let $\mathcal{A}$ and $\mathcal{B}$ be Banach algebras and let $T: \mathcal{B} \rightarrow \mathcal{A}$ be a continuous homomorphism. Recently, we introduced a product $\mathcal{M}:=\mathcal{A} \times_{T} \mathcal{B}$, which is a strongly splitting Banach algebra extension of $\mathcal{B}$ by $\mathcal{A}$. In the present paper, we characterize biprojectivity, approximate biprojectivity and biflatness of $\mathcal{A} \times_{T} \mathcal{B}$ in terms of $\mathcal{A}$ and $\mathcal{B}$. We also study some notions of amenability such as approximate amenability and pseudo-amenability of $\mathcal{A} \times_{T} \mathcal{B}$.
\end{abstract}

\section{INTRODUCTION AND PRELIMINARIES}

Let $\mathcal{A}$ and $\mathcal{B}$ be Banach algebras and let $T: \mathcal{B} \rightarrow \mathcal{A}$ be a continuous homomorphism with $\|T\| \leq 1$. The $T$-Lau product $\mathcal{A} \times_{T} \mathcal{B}$ is defined as the Cartesian product $\mathcal{A} \times \mathcal{B}$ with the algebra product

$$
(a, b)\left(a^{\prime}, b^{\prime}\right)=\left(a a^{\prime}+T(b) a^{\prime}+a T\left(b^{\prime}\right), b b^{\prime}\right),
$$

and the norm $\|(a, b)\|=\|a\|+\|b\|$. This type of product was first introduced by Bhatt and Dabhi [5] for the case where $\mathcal{A}$ is commutative and was extended by the authors for the general case [12].

Here it should be noted that, our motivation of the study presented in this paper and previous paper [12] comes from some resent developments of the splitting of Banach algebra extensions which have been studied by many authors such as Bade et al. [1, 2, 3, 4], Feldman [7], Helemskii [11], Johnson [13, 14], Lau [16] and

Date: Received: Jul. 7, 2014; Accepted: Aug. 11, 2014.

* Corresponding author.

2010 Mathematics Subject Classification. Primary 43A07; Secondary 46H25.

Key words and phrases. Biprojectivity, approximate biprojectivity, biflatness, approximate amenability, pseudo-amenability. 
Monfared [17, 18]. Moreover, splitting of Banach algebra extensions has been a major tool in the study of Banach algebras. For example, module extensions as generalizations of Banach algebra extensions were introduced and first studied by Gourdeau [10] were used to show that amenability of $\mathcal{A}^{* *}$, the second dual space of $\mathcal{A}$, implies amenability of $\mathcal{A}$ and Zhang [22] used module extensions to answer an open problem regarding weak amenability, raised by Dales, Ghahramani and Grønbæk [6]. On the other hand, $\mathcal{A} \times_{T} \mathcal{B}$ is a strongly splitting Banach algebra extension of $\mathcal{B}$ by $\mathcal{A}$ that exhibits many properties that are not shared by arbitrary strongly splitting extensions. For example, commutativity is not preserved by a general strongly splitting extension whereas $\mathcal{A} \times_{T} \mathcal{B}$ is commutative if and only if the commutativity assumption are satisfied by $\mathcal{A}$ and $\mathcal{B}$. In addition to all these, the $T$-Lau product would provide not only new examples of Banach algebras by themselves, but also it provide a wealth of (counter) examples in different branches of functional analysis. These facts suggest that $T$-Lau products are worth to study.

In [12], apart from the study of many basic properties of $\mathcal{A} \times_{T} \mathcal{B}$ such as characterization of bounded approximate identity, ideal structure, $n$-weak amenability and Arens regularity, useful characterizations of derivations from $\mathcal{A} \times_{T} \mathcal{B}$ into $\left(\mathcal{A} \times_{T} \mathcal{B}\right)^{(n)}$, the $n$th dual space of $\mathcal{A} \times_{T} \mathcal{B}$, were given. In continuation of our study on the $T$-Lau product of Banach algebras, in the present paper, we characterize some homological notions of $\mathcal{A} \times_{T} \mathcal{B}$ in terms of $\mathcal{A}$ and $\mathcal{B}$. Finally, some notions of amenability such as pseudo-amenability and approximate amenability, are investigated in detail for the $T$-Lau product of Banach algebras.

\section{Biprojectivity and Biflatness of $\mathcal{A} \times_{T} \mathcal{B}$}

In what follows, $\mathcal{A}$ and $\mathcal{B}$ are Banach algebras and $T: \mathcal{B} \rightarrow \mathcal{A}$ is a homomorphism with $\|T\| \leq 1$. The notation $\mathcal{M}$ is used to denote the $T$-Lau product of the Banach algebras $\mathcal{A}$ and $\mathcal{B}, r_{\mathcal{A}}$ denotes the mapping defined by $r_{\mathcal{A}}((a, b))=a+T(b)$ from $\mathcal{M}$ into $\mathcal{A}$, and $s_{\mathcal{B}}: \mathcal{B} \rightarrow \mathcal{M}$ denotes the map defined by the equality $s_{\mathcal{B}}(b)=(-T(b), b)$ for all $b \in \mathcal{B}$. Moreover, $\iota_{\mathcal{A}}$ denotes the usual injection map from $\mathcal{A}$ into $\mathcal{M}$ and $q_{\mathcal{B}}$ denotes the usual projection map from $\mathcal{M}$ into $\mathcal{B}$. Let also, for each Banach algebra $\mathcal{A}, \pi_{\mathcal{A}}$ denote the product morphism from the projective tensor product $\mathcal{A} \widehat{\otimes} \mathcal{A}$ into $\mathcal{A}$. A routine computations show that $\mathcal{A} \widehat{\otimes} \mathcal{A}$ becomes a Banach $\mathcal{A}$-bimodule with the following actions:

$$
a \cdot(b \otimes c)=a b \otimes c, \quad(b \otimes c) \cdot a=b \otimes c a
$$

for all $a, b, c \in \mathcal{A}$. With the above actions $\pi_{\mathcal{A}}$ becomes an $\mathcal{A}$-bimodule homomorphism. In what follows the notation $B(\mathcal{A}, \mathcal{A} \widehat{\otimes} \mathcal{A})$ is used into denote the space of all bounded linear operators from $\mathcal{A}$ into $\mathcal{A} \widehat{\otimes} \mathcal{A}$.

Recall that a Banach algebra $\mathcal{A}$ is said to be biprojective if there is a bounded $\mathcal{A}$-bimodule homomorphism $\rho_{\mathcal{A}}: \mathcal{A} \rightarrow \mathcal{A} \widehat{\otimes} \mathcal{A}$, such that $\pi_{\mathcal{A}} \circ \rho_{\mathcal{A}}=i d_{\mathcal{A}}$. Also, $\mathcal{A}$ is called biflat if there is a bounded $\mathcal{A}$-bimodule homomorphism $\theta_{\mathcal{A}}:(\mathcal{A} \widehat{\otimes} \mathcal{A})^{*} \rightarrow \mathcal{A}^{*}$ such that $\theta_{\mathcal{A}} \circ\left(\pi_{\mathcal{A}}\right)^{*}=i d_{\mathcal{A}^{*}}$. We also recall from [21] that $\mathcal{A}$ is approximately biprojective if there is a net $\left(\rho_{\alpha}^{\mathcal{A}}\right)$ of bounded $\mathcal{A}$-bimodule homomorphisms from $\mathcal{A}$ into $\mathcal{A} \widehat{\otimes} \mathcal{A}$ such that $\pi_{\mathcal{A}} \circ \rho_{\alpha}^{\mathcal{A}}(a) \rightarrow a$ for all $a \in \mathcal{A}$. Following Pirkovskii [19] 
for two Banach $\mathcal{A}$-bimodules $X$ and $Y$, a net $\left(\rho_{\alpha}\right)$ of bounded linear maps from $X$ into $Y$, satisfying

$$
\rho_{\alpha}(a \cdot x)-a \cdot \rho_{\alpha}(x) \rightarrow 0, \quad \rho_{\alpha}(x \cdot a)-\rho_{\alpha}(x) \cdot a \rightarrow 0
$$

for all $a \in \mathcal{A}$, will be called an approximate $\mathcal{A}$-bimodule morphism from $X$ into $Y$. Recently, a new notion of approximate biprojectivity introduced and studied by Pourmahmoud-Aghababa [20], which is based on approximate $\mathcal{A}$-bimodule morphisms. Precisely, in the sense of [20], a Banach algebra $\mathcal{A}$ is called approximately biprojective if there exists an approximate $\mathcal{A}$-bimodule morphism $\left(\rho_{\alpha}^{\mathcal{A}}\right)$ from $\mathcal{A}$ into $\mathcal{A} \widehat{\otimes} \mathcal{A}$ such that $\pi_{\mathcal{A}} \circ \rho_{\alpha}^{\mathcal{A}}(a) \longrightarrow a$ for all $a \in \mathcal{A}$.

Lemma 2.1. With the above notations, the following statements hold.

(i) $r_{\mathcal{A}} \circ \pi_{\mathcal{M}}=\pi_{\mathcal{A}} \circ\left(r_{\mathcal{A}} \otimes r_{\mathcal{A}}\right)$,

(ii) $\iota_{\mathcal{A}} \circ \pi_{\mathcal{A}}=\pi_{\mathcal{M}} \circ\left(\iota_{\mathcal{A}} \otimes \iota_{\mathcal{A}}\right)$,

(iii) $s_{\mathcal{B}} \circ \pi_{\mathcal{B}}=\pi_{\mathcal{M}} \circ\left(s_{\mathcal{B}} \otimes s_{\mathcal{B}}\right)$,

(iv) $q_{\mathcal{B}} \circ \pi_{\mathcal{M}}=\pi_{\mathcal{B}} \circ\left(q_{\mathcal{B}} \otimes q_{\mathcal{B}}\right)$.

Moreover, for all $a, a^{\prime}, a^{\prime \prime} \in \mathcal{A}$ and $b, b^{\prime}, b^{\prime \prime} \in \mathcal{B}$ we have

$(\mathrm{v})(a, b) \cdot\left(s_{\mathcal{B}} \otimes s_{\mathcal{B}}\right)\left(b^{\prime} \otimes b^{\prime \prime}\right)=\left(s_{\mathcal{B}} \otimes s_{\mathcal{B}}\right)\left(b b^{\prime} \otimes b^{\prime \prime}\right)$,

(vi) $\left(s_{\mathcal{B}} \otimes s_{\mathcal{B}}\right)\left(b^{\prime} \otimes b^{\prime \prime}\right) \cdot(a, b)=\left(s_{\mathcal{B}} \otimes s_{\mathcal{B}}\right)\left(b^{\prime} \otimes b^{\prime \prime} b\right)$,

(vii) $(a, b) \cdot\left(\iota_{\mathcal{A}} \otimes \iota_{\mathcal{A}}\right)\left(a^{\prime} \otimes a^{\prime \prime}\right)=\left(\iota_{\mathcal{A}} \otimes \iota_{\mathcal{A}}\right)\left([a+T(b)] a^{\prime} \otimes a^{\prime \prime}\right)$,

(viii) $\left(\iota_{\mathcal{A}} \otimes \iota_{\mathcal{A}}\right)\left(a^{\prime} \otimes a^{\prime \prime}\right) \cdot(a, b)=\left(\iota_{\mathcal{A}} \otimes \iota_{\mathcal{A}}\right)\left(a^{\prime} \otimes a^{\prime \prime}[a+T(b)]\right)$.

Proof. We prove the assertions (i), (v) and (vii). The proof of the other assertions are similar.

(i). Let $(a, b)$ and $\left(a^{\prime}, b^{\prime}\right)$ are arbitrary elements of $\mathcal{M}$. On the one hand, observe that

$$
\begin{aligned}
r_{\mathcal{A}} \circ \pi_{\mathcal{M}}\left((a, b) \otimes\left(a^{\prime}, b^{\prime}\right)\right) & =r_{\mathcal{A}}\left(a a^{\prime}+T(b) a^{\prime}+a T\left(b^{\prime}\right), b b^{\prime}\right) \\
& =a a^{\prime}+T(b) a^{\prime}+a T\left(b^{\prime}\right)+T\left(b b^{\prime}\right),
\end{aligned}
$$

and, on the other hand

$$
\begin{aligned}
\pi_{\mathcal{A}} \circ\left(r_{\mathcal{A}} \otimes r_{\mathcal{A}}\right)\left((a, b) \otimes\left(a^{\prime}, b^{\prime}\right)\right) & =\pi_{\mathcal{A}}\left([a+T(b)] \otimes\left[a^{\prime}+T\left(b^{\prime}\right)\right]\right) \\
& =a a^{\prime}+T(b) a^{\prime}+a T\left(b^{\prime}\right)+T\left(b b^{\prime}\right) .
\end{aligned}
$$

This completes the proof of (i).

(v). This is because of

$$
(a, b) \cdot\left[\left(-T\left(b^{\prime}\right), b^{\prime}\right) \otimes\left(-T\left(b^{\prime \prime}\right), b^{\prime \prime}\right)\right]=\left(-T\left(b b^{\prime}\right), b b^{\prime}\right) \otimes\left(-T\left(b^{\prime \prime}\right), b^{\prime \prime}\right),
$$

for all $a \in \mathcal{A}$ and $b, b^{\prime}, b^{\prime \prime} \in \mathcal{B}$.

(vi). This is because of

$$
\begin{aligned}
(a, b) \cdot\left[\left(a^{\prime}, 0\right) \otimes\left(a^{\prime \prime}, 0\right)\right] & =(a, b)\left(a^{\prime}, 0\right) \otimes\left(a^{\prime \prime}, 0\right) \\
& =\left(a+T(b) a^{\prime}, 0\right) \otimes\left(a^{\prime \prime}, 0\right),
\end{aligned}
$$

for all $a, a^{\prime}, a^{\prime \prime} \in \mathcal{A}$ and $b \in \mathcal{B}$.

In the following result we give the proof for the approximate biprojectivity in the sense of [20]. The proof for the other cases are similar.

Theorem 2.2. Let $\mathcal{M}$ be biprojective/approximately biprojective. Then $\mathcal{A}$ and $\mathcal{B}$ are biprojective/approximately biprojective. 
Proof. Denote the approximate right inverse maps given in the hypothesis by $\left(\rho_{\alpha}^{\mathcal{M}}\right)$. Now, for each $\alpha$ set

$$
\rho_{\alpha}^{\mathcal{B}}:=\left(q_{\mathcal{B}} \otimes q_{\mathcal{B}}\right) \circ \rho_{\alpha}^{\mathcal{M}} \circ \iota_{\mathcal{B}} \quad \text { and } \quad \rho_{\alpha}^{\mathcal{A}}:=\left(r_{\mathcal{A}} \otimes r_{\mathcal{A}}\right) \circ \rho_{\alpha}^{\mathcal{M}} \circ \iota_{\mathcal{A}} .
$$

Using Lemma 2.1 (i) and (iv) we can see that

$$
\pi_{\mathcal{A}} \circ \rho_{\alpha}^{\mathcal{A}}(a) \longrightarrow a
$$

and

$$
\pi_{\mathcal{B}} \circ \rho_{\alpha}^{\mathcal{B}}(b) \longrightarrow b .
$$

On the other hand, a direct computation shows that

$$
\begin{aligned}
\lim _{\alpha}\left[\rho_{\alpha}^{\mathcal{A}}\left(a a^{\prime}\right)-a \cdot \rho_{\alpha}^{\mathcal{A}}\left(a^{\prime}\right)\right] & =\left(r_{\mathcal{A}} \otimes r_{\mathcal{A}}\right)\left(\lim _{\alpha} \rho_{\alpha}^{\mathcal{M}}\left((a, 0)\left(a^{\prime}, 0\right)\right)-(a, 0) \cdot \rho_{\alpha}^{\mathcal{A}}\left(\left(a^{\prime}, 0\right)\right)\right) \\
\lim _{\alpha}\left[\rho_{\alpha}^{\mathcal{A}}\left(a a^{\prime}\right)-\rho_{\alpha}^{\mathcal{A}}(a) \cdot a^{\prime}\right] & =\left(r_{\mathcal{A}} \otimes r_{\mathcal{A}}\right)\left(\lim _{\alpha} \rho_{\alpha}^{\mathcal{M}}\left((a, 0)\left(a^{\prime}, 0\right)\right)-\rho_{\alpha}^{\mathcal{A}}((a, 0)) \cdot\left(a^{\prime}, 0\right)\right), \\
\lim _{\alpha}\left[\rho_{\alpha}^{\mathcal{B}}\left(b b^{\prime}\right)-b \cdot \rho_{\alpha}^{\mathcal{B}}\left(b^{\prime}\right)\right]=\left(q_{\mathcal{B}} \otimes q_{\mathcal{B}}\right)\left(\lim _{\alpha} \rho_{\alpha}^{\mathcal{M}}\left((0, b)\left(0, b^{\prime}\right)\right)-(0, b) \cdot \rho_{\alpha}^{\mathcal{B}}\left(\left(0, b^{\prime}\right)\right)\right), & (2.5) \\
\lim _{\alpha}\left[\rho_{\alpha}^{\mathcal{B}}\left(b b^{\prime}\right)-\rho_{\alpha}^{\mathcal{B}}(b) \cdot b^{\prime}\right] & =\left(q_{\mathcal{B}} \otimes q_{\mathcal{B}}\right)\left(\lim _{\alpha} \rho_{\alpha}^{\mathcal{M}}\left((0, b)\left(0, b^{\prime}\right)\right)-\rho_{\alpha}^{\mathcal{B}}((0, b)) \cdot\left(0, b^{\prime}\right)\right) .
\end{aligned}
$$

Now, invoke (2.1), (2.3) and (2.4) to conclude that $\mathcal{A}$ is approximately biprojective. Also (2.2), (2.5) and (2.6) imply that $\mathcal{B}$ is approximately biprojective.

Now we give sufficient conditions for the biprojectivity/approximate biprojectivity of $T$-Lau product of Banach algebras.

Theorem 2.3. Let $\mathcal{A}$ and $\mathcal{B}$ be biprojective/approximately biprojective Banach algebras. Then $\mathcal{M}$ is biprojective/approximately biprojective.

Proof. Let $\left(\rho_{\alpha}^{\mathcal{A}}\right)_{\alpha \in I} \subseteq B(\mathcal{A}, \mathcal{A} \widehat{\otimes} \mathcal{A})$ and $\left(\rho_{\beta}^{\mathcal{B}}\right)_{\beta \in J} \subseteq B(\mathcal{B}, \mathcal{B} \widehat{\otimes} \mathcal{B})$ respectively be approximate $\mathcal{A}$-bimodule and approximate $\mathcal{B}$-bimodule morphisms such that

$$
\pi_{\mathcal{A}} \circ \rho_{\alpha}^{\mathcal{A}}(a) \longrightarrow a, \quad \pi_{\mathcal{B}} \circ \rho_{\beta}^{\mathcal{B}}(b) \longrightarrow b
$$

for all $a \in \mathcal{A}$ and $b \in \mathcal{B}$. Now, consider the directed set

$$
\Gamma:=\{\gamma=(\alpha, \beta): \alpha \in I, \beta \in J\},
$$

with the order

$$
(\alpha, \beta) \preceq\left(\alpha^{\prime}, \beta^{\prime}\right) \Longleftrightarrow \alpha \leq \alpha^{\prime}, \beta \leq \beta^{\prime} .
$$

Then the net $\left(\rho_{\gamma}^{\mathcal{M}}\right) \subseteq B(\mathcal{M}, \mathcal{M} \widehat{\otimes} \mathcal{M})$ defined by

$\rho_{\gamma}^{\mathcal{M}}((a, b))=\left(\left(\iota_{\mathcal{A}} \otimes \iota_{\mathcal{A}}\right) \circ \rho_{\alpha}^{\mathcal{A}} \circ r_{\mathcal{A}}+\left(s_{\mathcal{B}} \otimes s_{\mathcal{B}}\right) \circ \rho_{\beta}^{\mathcal{B}} \circ q_{\mathcal{B}}\right)((a, b)), \quad((a, b) \in \mathcal{M})$ 
is as required for the approximate biprojectivity of $\mathcal{M}$. Indeed, for each $(a, b) \in$ $\mathcal{M}$ we have

$$
\begin{aligned}
& \pi_{\mathcal{M}} \circ \rho_{\gamma}^{\mathcal{M}}((a, b)) \\
= & \left(\pi_{\mathcal{M}} \circ\left(\iota_{\mathcal{A}} \otimes \iota_{\mathcal{A}}\right) \circ \rho_{\alpha}^{\mathcal{A}} \circ r_{\mathcal{A}}+\pi_{\mathcal{M}} \circ\left(s_{\mathcal{B}} \otimes s_{\mathcal{B}}\right) \circ \rho_{\beta}^{\mathcal{B}} \circ q_{\mathcal{B}}\right)((a, b)) \\
= & \left(\iota_{\mathcal{A}} \circ \pi_{\mathcal{A}} \circ \rho_{\alpha}^{\mathcal{A}} \circ r_{\mathcal{A}}+s_{\mathcal{B}} \circ \pi_{\mathcal{B}} \circ \rho_{\beta}^{\mathcal{B}} \circ q_{\mathcal{B}}\right)((a, b)) \\
\longrightarrow & \left(\iota_{\mathcal{A}} \circ r_{\mathcal{A}}+s_{\mathcal{B}} \circ q_{\mathcal{B}}\right)((a, b)) \\
= & (a+T(b), 0)+(-T(b), b)=(a, b),
\end{aligned}
$$

where the second equality follows from the Lemma 2.1 (ii) and (iii). Thus, for each $(a, b) \in \mathcal{M}$ we have

$$
\pi_{\mathcal{M}} \circ \rho_{\gamma}^{\mathcal{M}}((a, b)) \longrightarrow(a, b) .
$$

Moreover, using Lemma 2.1 (v) and (vii) we have

$$
\rho_{\gamma}^{\mathcal{M}}\left((a, b)\left(a^{\prime}, b^{\prime}\right)\right)-(a, b) \cdot \rho_{(\alpha, \beta)}^{\mathcal{M}}\left(\left(a^{\prime}, b^{\prime}\right)\right) \longrightarrow 0 .
$$

By a same argument as above we have

$$
\rho_{\gamma}^{\mathcal{M}}\left((a, b)\left(a^{\prime}, b^{\prime}\right)\right)-\rho_{\gamma}^{\mathcal{M}}((a, b)) \cdot\left(a^{\prime}, b^{\prime}\right) \longrightarrow 0,
$$

and this completes the proof.

Remark 2.4. All of our results in Theorems 2.2 and 2.3 can be proved, by slight modifications of the arguments, with "approximate biprojectivity in the sense of [20]" replaced by "approximate biprojectivity in the sense of [21]".

In the following our aim is to study the biflatness of $T$-Lau product of Banach algebras. In fact, we will show that $\mathcal{A}$ and $\mathcal{B}$ are biflat if and only if $\mathcal{M}$ is biflat.

Theorem 2.5. Let $\mathcal{M}$ be biflat. Then $\mathcal{A}$ and $\mathcal{B}$ are biflat.

Proof. Let $\theta_{\mathcal{M}}$ be a bounded left inverse for $\left(\pi_{\mathcal{M}}\right)^{*}$ which is a $\mathcal{M}$-bimodule homomorphism. Define $\theta_{\mathcal{B}}:(\mathcal{B} \widehat{\otimes} \mathcal{B})^{*} \rightarrow \mathcal{B}^{*}$ and $\theta_{\mathcal{A}}:(\mathcal{A} \widehat{\otimes} \mathcal{A})^{*} \rightarrow \mathcal{A}^{*}$ by $\theta_{\mathcal{B}}=$ $\left(\iota_{B}\right)^{*} \circ \theta_{M} \circ\left(q_{\mathcal{B}} \otimes q_{\mathcal{B}}\right)^{*}$ and $\theta_{\mathcal{A}}=\left(\iota_{\mathcal{A}}\right)^{*} \circ \theta_{M} \circ\left(r_{\mathcal{A}} \otimes r_{\mathcal{A}}\right)^{*}$, respectively. Obviously $\theta_{\mathcal{B}}$ and $\theta_{\mathcal{A}}$ are bounded $\mathcal{B}$-bimodule and $\mathcal{A}$-bimodule homomorphisms, respectively. Moreover,

$$
\begin{aligned}
\theta_{\mathcal{A}} \circ\left(\pi_{\mathcal{A}}\right)^{*} & =\left(\left(\iota_{\mathcal{A}}\right)^{*} \circ \theta_{M} \circ\left(r_{\mathcal{A}} \otimes r_{\mathcal{A}}\right)^{*}\right) \circ\left(\pi_{\mathcal{A}}\right)^{*} \\
& =\left(\iota_{\mathcal{A}}\right)^{*} \circ \theta_{M} \circ\left(\left(r_{\mathcal{A}} \otimes r_{\mathcal{A}}\right)^{*} \circ\left(\pi_{\mathcal{A}}\right)^{*}\right) \\
& =\left(\iota_{\mathcal{A}}\right)^{*} \circ \theta_{M} \circ\left(\left(\pi_{\mathcal{M}}\right)^{*} \circ\left(r_{\mathcal{A}}\right)^{*}\right) \\
& =\left(\iota_{\mathcal{A}}\right)^{*} \circ\left(\theta_{M} \circ\left(\pi_{\mathcal{M}}\right)^{*}\right) \circ\left(r_{\mathcal{A}}\right)^{*} \\
& =\left(\iota_{\mathcal{A}}\right)^{*} \circ\left(\theta_{M} \circ\left(\pi_{\mathcal{M}}\right)^{*}\right) \circ\left(r_{\mathcal{A}}\right)^{*} \\
& =\left(\iota_{\mathcal{A}}\right)^{*} \circ i d_{\mathcal{M}^{*}} \circ\left(r_{\mathcal{A}}\right)^{*} \\
& =\left(r_{\mathcal{A}} \circ \iota_{\mathcal{A}}\right)^{*} \\
& =i d_{\mathcal{A}^{*}},
\end{aligned}
$$

where the third equality follows from Lemma 2.1. Using the same proof as above, we can show that $\theta_{\mathcal{B}} \circ\left(\pi_{\mathcal{B}}\right)^{*}=i d_{\mathcal{B}^{*}}$, as required. 
Now we consider the converse of Theorem 2.5. Before proceeding further, let us mention that $\left(\mathcal{A} \times_{T} \mathcal{B}\right)^{*}$, the first dual space of $\mathcal{A} \times_{T} \mathcal{B}$, can be identified with $\mathcal{A}^{*} \times \mathcal{B}^{*}$ with the following definition

$$
\langle(f, g),(a, b)\rangle=\langle f, a\rangle+\langle g, b\rangle,
$$

for all $a \in \mathcal{A}, b \in \mathcal{B}, f \in \mathcal{A}^{*}$ and $g \in \mathcal{B}^{*}$. Moreover, we can find that the $\left(A \times_{T} B\right)$-bimodule actions on $\left(A \times_{T} B\right)^{*}$ are formulated as follows

$$
(a, b) \cdot(f, g)=\left([a+T(b)] \cdot f, T^{*}(a \cdot f)+b \cdot g\right)
$$

and

$$
(f, g) \cdot(a, b)=\left(f \cdot[a+T(b)], T^{*}(f \cdot a)+g \cdot b\right)
$$

for all $(a, b) \in A \times_{T} B$ and $(f, g) \in A^{*} \times B^{*}$, where $T^{*}$ is the adjoint of the operator $T$.

Theorem 2.6. Let $\mathcal{A}$ and $\mathcal{B}$ be biflat. Then $\mathcal{M}$ is biflat.

Proof. Let $\theta_{\mathcal{A}}:(\mathcal{A} \widehat{\otimes} \mathcal{A})^{*} \rightarrow \mathcal{A}^{*}$ be a bounded $\mathcal{A}$-bimodule homomorphism and let $\theta_{\mathcal{B}}:(\mathcal{B} \widehat{\otimes} \mathcal{B})^{*} \rightarrow \mathcal{B}^{*}$ be a bounded $\mathcal{B}$-bimodule homomorphism such that $\theta_{\mathcal{A}} \circ$ $\left(\pi_{\mathcal{A}}\right)^{*}=i d_{\mathcal{A}^{*}}$ and $\theta_{\mathcal{B}} \circ\left(\pi_{\mathcal{B}}\right)^{*}=i d_{\mathcal{B}^{*}}$. Define the map $\theta_{\mathcal{M}}:(\mathcal{M} \widehat{\otimes} \mathcal{M})^{*} \rightarrow \mathcal{M}^{*}$ by

$$
\begin{aligned}
\theta_{\mathcal{M}}(\Phi) & =\left(\theta_{\mathcal{A}} \circ\left(\iota_{\mathcal{A}} \otimes \iota_{\mathcal{A}}\right)^{*}(\Phi), \theta_{\mathcal{B}} \circ\left(s_{\mathcal{B}} \otimes s_{\mathcal{B}}\right)^{*}(\Phi)\right. \\
& \left.+T^{*}\left(\theta_{\mathcal{A}} \circ\left(\iota_{\mathcal{A}} \otimes \iota_{\mathcal{A}}\right)^{*}(\Phi)\right)\right)
\end{aligned}
$$

for all $\Phi \in(\mathcal{M} \widehat{\otimes} \mathcal{M})^{*}$. Then for each $(f, g) \in \mathcal{A}^{*} \times \mathcal{B}^{*}$ we have

$$
\begin{aligned}
\left\langle\theta_{\mathcal{M}} \circ\right. & \left.\left(\pi_{\mathcal{M}}\right)^{*},(f, g)\right\rangle \\
& =\left(\theta_{\mathcal{A}} \circ\left(\iota_{\mathcal{A}} \otimes \iota_{\mathcal{A}}\right)^{*} \circ\left(\pi_{\mathcal{M}}\right)^{*}((f, g)), \theta_{\mathcal{B}} \circ\left(s_{\mathcal{B}} \otimes s_{\mathcal{B}}\right)^{*} \circ\left(\pi_{\mathcal{M}}\right)^{*}((f, g))\right. \\
& \left.+T^{*}\left(\theta_{\mathcal{A}} \circ\left(\iota_{\mathcal{A}} \otimes \iota_{\mathcal{A}}\right)^{*} \circ\left(\pi_{\mathcal{M}}\right)^{*}((f, g))\right)\right) \\
& =\left(\theta_{\mathcal{A}} \circ\left(\pi_{\mathcal{A}}\right)^{*} \circ\left(\iota_{\mathcal{A}}\right)^{*}((f, g)), \theta_{\mathcal{B}} \circ\left(\pi_{\mathcal{B}}\right)^{*} \circ\left(s_{\mathcal{B}}\right)^{*}((f, g))\right. \\
& \left.+T^{*}\left(\theta_{\mathcal{A}} \circ\left(\pi_{\mathcal{A}}\right)^{*} \circ\left(\iota_{\mathcal{A}}\right)^{*}((f, g))\right)\right) \\
& =\left(i d_{\mathcal{A}^{*}} \circ\left(\iota_{\mathcal{A}}\right)^{*}(\Phi), i d_{\mathcal{B}^{*}} \circ\left(s_{\mathcal{B}}\right)^{*}((f, g))\right. \\
& \left.+T^{*}\left(i d_{\mathcal{A}^{*}} \circ\left(\iota_{\mathcal{A}}\right)^{*}((f, g))\right)\right) \\
& =\left(f,\left(-T^{*}(f)+g\right)+T^{*}(f)\right) \\
& =(f, g)
\end{aligned}
$$

where the second equality follows from Lemma 2.1. Thus $\theta_{\mathcal{M}} \circ\left(\pi_{\mathcal{M}}\right)^{*}=i d_{\mathcal{M}^{*}}$. Now, we show that $\theta_{\mathcal{M}}$ is a $\mathcal{M}$-bimodule homomorphism. To see this, first note that it is easy to check that the following equalities hold.

$$
\begin{aligned}
& \left(\iota_{\mathcal{A}} \otimes \iota_{\mathcal{A}}\right)^{*}(\Phi \cdot(a, b))=\left(\iota_{\mathcal{A}} \otimes \iota_{\mathcal{A}}\right)^{*}(\Phi) \cdot[a+T(b)], \\
& \left(\iota_{\mathcal{A}} \otimes \iota_{\mathcal{A}}\right)^{*}((a, b) \cdot \Phi)=[a+T(b)] \cdot\left(\iota_{\mathcal{A}} \otimes \iota_{\mathcal{A}}\right)^{*}(\Phi), \\
& \left(s_{\mathcal{B}} \otimes s_{\mathcal{B}}\right)^{*}(\Phi \cdot(a, b))=\left(s_{\mathcal{B}} \otimes s_{\mathcal{B}}\right)^{*}(\Phi) \cdot b, \\
& \left(s_{\mathcal{B}} \otimes s_{\mathcal{B}}\right)^{*}((a, b) \cdot \Phi)=b \cdot\left(s_{\mathcal{B}} \otimes s_{\mathcal{B}}\right)^{*}(\Phi) .
\end{aligned}
$$


for all $\Phi \in(\mathcal{M} \widehat{\otimes} \mathcal{M})^{*}$ and $(a, b) \in \mathcal{M}$. Thus, if we set $f:=\theta_{\mathcal{A}} \circ\left(\iota_{\mathcal{A}} \otimes \iota_{\mathcal{A}}\right)^{*}(\Phi)$ and $g:=\theta_{\mathcal{B}} \circ\left(s_{\mathcal{B}} \otimes s_{\mathcal{B}}\right)^{*}(\Phi)$, then we have

$$
\begin{aligned}
\theta_{\mathcal{M}}(\Phi \cdot(a, b)) & =\left(\theta_{\mathcal{A}} \circ\left(\iota_{\mathcal{A}} \otimes \iota_{\mathcal{A}}\right)^{*}(\Phi \cdot(a, b)), \theta_{\mathcal{B}} \circ\left(s_{\mathcal{B}} \otimes s_{\mathcal{B}}\right)^{*}(\Phi \cdot(a, b))\right. \\
& \left.+T^{*}\left(\theta_{\mathcal{A}} \circ\left(\iota_{\mathcal{A}} \otimes \iota_{\mathcal{A}}\right)^{*}(\Phi \cdot(a, b))\right)\right) \\
& =\left(f \cdot[a+T(b)], g \cdot b+T^{*}(f \cdot[a+T(b)])\right) \\
& =\left(f \cdot[a+T(b)],\left[g+T^{*}(f)\right] \cdot b+T^{*}(f \cdot a)\right) \\
& =\left(f, g+T^{*}(f)\right) \cdot(a, b) \\
& =\theta_{\mathcal{M}}(\Phi) \cdot(a, b) .
\end{aligned}
$$

Using the same proof as above, we can show that $\theta_{\mathcal{M}}((a, b) \cdot \Phi)=(a, b) \cdot \theta_{\mathcal{M}}(\Phi)$. Thus $\mathcal{M}$ is biflat.

\section{Some notions of amenability on $\mathcal{A} \times_{T} \mathcal{B}$}

Let $\mathcal{A}$ be a Banach algebra and let $X$ be a Banach $\mathcal{A}$-bimodule. A derivation from $\mathcal{A}$ into $X$ is a linear map $D: \mathcal{A} \rightarrow X$ such that

$$
D(a b)=a \cdot D(b)+D(a) \cdot b,
$$

for all $a, b \in \mathcal{A}$. For example, the $\operatorname{map} \operatorname{ad}_{x}: \mathcal{A} \rightarrow X$ defined by $\operatorname{ad}_{x}(a)=a \cdot x-x \cdot a$ is a derivation for all $a \in \mathcal{A}$ and $x \in X$. Such derivations are called inner derivations. Here, let us mention that $X^{*}$, the first dual space of $X$, becomes a Banach $\mathcal{A}$-bimodule in the natural way. The algebra $\mathcal{A}$ is called amenable if every continuous derivation $D: \mathcal{A} \rightarrow X^{*}$ is inner for each Banach $\mathcal{A}$-bimodule $X$, and is called contractible if every continuous derivation $D: \mathcal{A} \rightarrow X$ is inner for each Banach $\mathcal{A}$-bimodule $X$. A derivation $D: \mathcal{A} \rightarrow X$ is called approximately inner if there exists a net $\left(x_{\alpha}\right) \subseteq X$ such that $D(a)=\lim _{\alpha} \operatorname{ad}_{x_{\alpha}}(a)$ for all $a \in \mathcal{A}$. A Banach algebra $\mathcal{A}$ is approximately amenable if every continuous derivation $D: \mathcal{A} \rightarrow X^{*}$ is approximately inner. A Banach algebra $\mathcal{A}$ is pseudo-amenable if there is a net $\left(u_{\alpha}\right) \subseteq \mathcal{A} \widehat{\otimes} \mathcal{A}$, called an approximate diagonal, such that

$$
a \cdot u_{\alpha}-u_{\alpha} \cdot a \longrightarrow 0, \quad \pi_{\mathcal{A}}\left(u_{\alpha}\right) a \longrightarrow a
$$

for all $a \in \mathcal{A}$, and is pseudo-contractible if it has a central approximate diagonal, i.e. an approximate diagonal $\left(u_{\alpha}\right)$ satisfying $a \cdot u_{\alpha}=u_{\alpha} \cdot a$ for all $a \in \mathcal{A}$; see [9] for more details.

Remark 3.1. Let $\mathcal{A}$ be a Banach algebra with an idempotent element $e$ and let $\theta \in \Delta(\mathcal{B})$, the character space of the Banach algebra $\mathcal{B}$. Then it is easy to check that the map $T_{\theta}: \mathcal{B} \rightarrow \mathcal{A}$ defined by $T_{\theta}(b)=\theta(b) e$ is a continuous homomorphism. In this case if $e$ is an identity for $\mathcal{A}$, then $\mathcal{A} \times_{T_{\theta}} \mathcal{B}$ coincides with the $\theta$-Lau product $\mathcal{A} \times{ }_{\theta} \mathcal{B}$; see [17] for more details. In [15] the authors investigate biflatness and biprojectivity of $\mathcal{A} \times{ }_{\theta} \mathcal{B}$ in the case where $\mathcal{A}$ is unital. Thus, in the previous section we generalized these results to that of $T$-Lau product of Banach algebras.

As a result of Theorems 2.2, 2.3 and Remark 2.4 we have the following corollary for the approximate biprojectivity of $\mathcal{A} \times{ }_{\theta} \mathcal{B}$ and its relation with $\mathcal{A}$ and $\mathcal{B}$ in the sense of [21]. 
Corollary 3.2. Let $\mathcal{A}$ and $\mathcal{B}$ be two Banach algebras with $\theta \in \Delta(\mathcal{B})$. Then the following statements are equivalent.

(i) $\mathcal{A}$ is unital and $\mathcal{A} \times_{\theta} \mathcal{B}$ is approximately biprojective.

(ii) $\mathcal{A}$ is contractible and $\mathcal{B}$ is approximately biprojective.

Proof. (i) $\Rightarrow$ (ii). Suppose that $e$ is the identity element of $\mathcal{A}$. Then $\mathcal{A} \times_{T_{\theta}} \mathcal{B}$ coincides with $\mathcal{A} \times{ }_{\theta} \mathcal{B}$ as shown in above remark. Thus, approximate biprojectivity of $\mathcal{A} \times{ }_{\theta} \mathcal{B}$ implies that both $\mathcal{A}$ and $\mathcal{B}$ are approximately biprojective by Theorem 2.2 and Remark 2.4. Since $\mathcal{A}$ is unital it follows that it is contractible by $[9$, Theorem 2.4 and Proposition 3.8]. The implication (ii) $\Rightarrow$ (i) follows from Theorem 2.3 and Remark 2.4 with the fact that any contractible Banach algebra is unital and biprojective.

Proposition 3.3. $\mathcal{M}$ is pseudo-amenable/pseudo-contractible if and only if $\mathcal{A}$ and $\mathcal{B}$ are pseudo-amenable/pseudo-contractible.

Proof. We give the proof for the pseudo-amenable case. The proof for the other case is similar. Let $\mathcal{M}$ be pseudo-amenable. Then pseudo-amenability of $\mathcal{B}$ follows from [9, Proposition 2.2] together with the fact that the projection map $q_{\mathcal{B}}$ from $\mathcal{M}$ onto $\mathcal{B}$ is a continuous epimorphism. Now, we show that $\mathcal{A}$ is also pseudoamenable. By assumption, $\mathcal{M}$ has an approximate diagonal, say $\left(u_{\alpha}\right) \subseteq \mathcal{M} \widehat{\otimes} \mathcal{M}$. Now, consider the net $r_{\mathcal{A}} \otimes r_{\mathcal{A}}\left(u_{\alpha}\right)$. Observe that

$$
a \cdot\left(r_{\mathcal{A}} \otimes r_{\mathcal{A}}\right)\left(u_{\alpha}\right)-\left(r_{\mathcal{A}} \otimes r_{\mathcal{A}}\right)\left(u_{\alpha}\right) \cdot a=\left(r_{\mathcal{A}} \otimes r_{\mathcal{A}}\right)\left((a, 0) \cdot u_{\alpha}-u_{\alpha} \cdot(a, 0)\right),
$$

and

$$
\pi_{\mathcal{A}} \circ\left(r_{\mathcal{A}} \otimes r_{\mathcal{A}}\right)\left(u_{\alpha}\right)=r_{\mathcal{A}} \circ \pi_{\mathcal{M}}\left(u_{\alpha}\right)
$$

for all $a \in \mathcal{A}$ and $\alpha$, where the second equality follows from Lemma 2.1. These results imply that $\mathcal{A}$ is pseudo-amenable.

Conversely, suppose that both $\mathcal{A}$ and $\mathcal{B}$ are pseudo-amenable. Let $\left(u_{\alpha}\right)_{\alpha \in I} \subseteq$ $\mathcal{A} \widehat{\otimes} \mathcal{A}$ and $\left(u_{\beta}\right)_{\beta \in J} \subseteq \mathcal{B} \widehat{\otimes} \mathcal{B}$ be approximate diagonal for $\mathcal{A}$ and $\mathcal{B}$, respectively. Given the directed set

$$
\Gamma:=\{\gamma=(\alpha, \beta): \alpha \in I, \beta \in J\}
$$

with the order

$$
(\alpha, \beta) \preceq\left(\alpha^{\prime}, \beta^{\prime}\right) \Longleftrightarrow \alpha \leq \alpha^{\prime}, \beta \leq \beta^{\prime}
$$

Then the net $\left(u_{\gamma}\right) \subseteq \mathcal{M} \widehat{\otimes} \mathcal{M}$ defined by

$$
u_{\gamma}:=\left(\iota_{\mathcal{A}} \otimes \iota_{\mathcal{A}}\right)\left(u_{\alpha}\right)+\left(s_{\mathcal{B}} \otimes s_{\mathcal{B}}\right)\left(u_{\beta}\right)
$$

is an approximate diagonal for $\mathcal{M}$. In fact, by Lemma 2.1 we have

$$
\begin{aligned}
(a, b) \cdot u_{\gamma}-u_{\gamma} \cdot(a, b) & =\left(\iota_{\mathcal{A}} \otimes \iota_{\mathcal{A}}\right)\left([a+T(b)] \cdot u_{\alpha}-u_{\alpha} \cdot[a+T(b)]\right) \\
& +\left(s_{\mathcal{B}} \otimes s_{\mathcal{B}}\right)\left(b \cdot u_{\beta}-u_{\beta} \cdot b\right),
\end{aligned}
$$


and again Lemma 2.1 implies that

$$
\begin{aligned}
\pi_{\mathcal{M}}\left(u_{\gamma}\right)(a, b) & =\pi_{\mathcal{M}}\left(\left(\iota_{\mathcal{A}} \otimes \iota_{\mathcal{A}}\right)\left(u_{\alpha}\right)+\left(s_{\mathcal{B}} \otimes s_{\mathcal{B}}\right)\left(u_{\beta}\right)\right)(a, b) \\
& =\left[\iota_{\mathcal{A}} \circ \pi_{\mathcal{A}}\left(u_{\alpha}\right)+s_{\mathcal{B}} \circ \pi_{\mathcal{B}}\left(u_{\beta}\right)\right](a, b) \\
& =\left[\left(\pi_{\mathcal{A}}\left(u_{\alpha}\right), 0\right)+\left(-T\left(\pi_{\mathcal{B}}\left(u_{\beta}\right)\right), \pi_{\mathcal{B}}\left(u_{\beta}\right)\right)\right](a, b) \\
& =\left(\pi_{\mathcal{A}}\left(u_{\alpha}\right) a+\pi_{\mathcal{A}}\left(u_{\alpha}\right) T(b), 0\right) \\
& +\left(-T\left(\pi_{\mathcal{B}}\left(u_{\beta}\right)\right) T(b), \pi_{\mathcal{B}}\left(u_{\beta}\right) b\right) \\
& \longrightarrow(a+T(b), 0)+(-T(b), b)=(a, b)
\end{aligned}
$$

for all $(a, b) \in \mathcal{M}$. Hence, $\mathcal{M}$ is pseudo-amenable.

Proposition 3.4. Let $\mathcal{M}$ be approximately amenable. Then $\mathcal{A}$ and $\mathcal{B}$ are approximately amenable.

Proof. Since $\mathcal{M} / \mathcal{A}$ is isometrically isomorphic to $\mathcal{B}$, approximate amenability of $\mathcal{M}$ implies that $\mathcal{B}$ is also approximately amenable by [8, Corollary 2.1]. Now, we show that $\mathcal{A}$ is also approximately amenable. Let $X$ be a Banach $\mathcal{A}$-bimodule and $D: \mathcal{A} \longrightarrow X^{*}$ be a continuous derivation. Then it is easy to show that $X$ is an $\left(\mathcal{A} \times_{T} \mathcal{B}\right)$-bimodule with the module actions

$$
(a, b) \cdot x=[a+T(b)] \cdot x, \quad x \cdot(a, b)=x \cdot[a+T(b)],
$$

for all $a \in \mathcal{A}, b \in \mathcal{B}$ and $x \in X$. We prove that the map

$$
\widetilde{D}: \mathcal{M} \longrightarrow X^{*}
$$

defined by $\widetilde{D}=D \circ r_{\mathcal{A}}$ is a derivation. In fact, for every $(a, b)$ and $\left(a^{\prime}, b^{\prime}\right)$ in $\mathcal{M}$ we have

$$
\begin{aligned}
\widetilde{D}\left((a, b)\left(a^{\prime}, b^{\prime}\right)\right) & =\widetilde{D}\left(\left(a a^{\prime}+T(b) a^{\prime}+a T\left(b^{\prime}\right), b b^{\prime}\right)\right) \\
& =D\left(a a^{\prime}\right)+D\left(T(b) a^{\prime}\right)+D\left(a T\left(b^{\prime}\right)\right)+D\left(T(b) T\left(b^{\prime}\right)\right) \\
& =a \cdot D\left(a^{\prime}\right)+D(a) \cdot a^{\prime}+T(b) D\left(a^{\prime}\right)+D(T(b)) a^{\prime}+D(a) T\left(\left(\mathbf{B}^{\prime} \mathbf{\$}\right)\right. \\
& +a \cdot D\left(T\left(b^{\prime}\right)\right)+T(b) \cdot D\left(T\left(b^{\prime}\right)\right)+D(T(b)) \cdot T\left(b^{\prime}\right) .
\end{aligned}
$$

On the other hand,

$$
\begin{aligned}
(a, b) \cdot \widetilde{D}\left(\left(a^{\prime}, b^{\prime}\right)\right) & =(a, b) \cdot D\left(a^{\prime}+T\left(b^{\prime}\right)\right) \\
& =[a+T(b)] \cdot\left[D\left(a^{\prime}\right)+D\left(T\left(b^{\prime}\right)\right)\right]
\end{aligned}
$$

and

$$
\begin{aligned}
\widetilde{D}((a, b)) \cdot\left(a^{\prime}, b^{\prime}\right) & =D(a+T(b)) \cdot\left(a^{\prime}, b^{\prime}\right) \\
& =[D(a)+D(T(b))] \cdot\left[a^{\prime}+T\left(b^{\prime}\right)\right]
\end{aligned}
$$

for each $a, a^{\prime} \in \mathcal{A}$ and $b, b^{\prime} \in \mathcal{B}$. Adding (3.5) to (3.7) and comparing with (3.1), we conclude that $\widetilde{D}$ is a derivation. From the approximate amenability of $A \times_{T} \mathcal{B}$, it follows that there is a net $\left(\xi_{\alpha}\right) \subseteq X^{*}$ such that $\widetilde{D}((a, b))=\lim _{\alpha} \operatorname{ad}_{\xi_{\alpha}}((a, b))$ for all $(a, b) \in \mathcal{M}$. We claim that $D(a)=\lim _{\alpha} \operatorname{ad}_{\xi_{\alpha}}(a)$ for all $a \in \mathcal{A}$; indeed,

$$
\begin{aligned}
D(a)=\widetilde{D}((a, 0)) & =\lim _{\alpha}\left[(a, 0) \cdot \xi_{\alpha}-\xi_{\alpha} \cdot(a, 0)\right] \\
& =\lim _{\alpha}\left[a \cdot \xi_{\alpha}-\xi_{\alpha} \cdot a\right]
\end{aligned}
$$


for all $a \in \mathcal{A}$, as required.

We do not know if the converse of the Proposition 3.4 is valid, however following results are a partial converse for the Proposition 3.4. Before we give the next result, recall that a Banach $\mathcal{A}$-bimodule $X$ is called neo-unital if

$$
X=\mathcal{A} \cdot X \cdot \mathcal{A}=\{a \cdot x \cdot a: a, b \in \mathcal{A}, x \in X\} .
$$

Proposition 3.5. Let $\mathcal{A}$ and $\mathcal{B}$ be approximately amenable Banach algebras. Then, for any neo-unital Banach $\mathcal{M}$-bimodule $X$, continuous derivations from $\mathcal{M}$ into $X^{*}$ are weak* approximately inner.

Proof. Let $X$ be a neo-unital Banach $\mathcal{M}$-bimodule and let $D: \mathcal{M} \rightarrow X^{*}$ be a continuous derivation. Then it is easy to check that $X^{*}$ is both $\mathcal{A}$-bimodule and $\mathcal{B}$-bimodule with the module actions

$$
a \cdot \xi=(a, 0) \cdot \xi, \quad \xi \cdot a=\xi \cdot(a, 0), \quad b \cdot \xi=(-T(b), b) \cdot \xi, \quad \xi \cdot b=\xi \cdot(-T(b), b)
$$

for all $a \in \mathcal{A}, b \in \mathcal{B}$ and $\xi \in X^{*}$. Now, consider the linear maps $D_{\mathcal{A}}: \mathcal{A} \rightarrow X^{*}$ and $D_{\mathcal{B}}: \mathcal{B} \rightarrow X^{*}$ defined by $D_{\mathcal{A}}(a)=D((a, 0))$ and $D_{\mathcal{B}}(b)=D((-T(b), b))$. Trivially $D_{\mathcal{A}}$ and $D_{\mathcal{B}}$ are continuous derivations. Thus by assumption there are nets $\left(\xi_{\gamma}\right)$ and $\left(\eta_{\gamma}\right)$ in $X^{*}$ such that

$$
\begin{aligned}
D_{\mathcal{A}}(a) & =D((a, 0))=\lim _{\gamma}\left[(a, 0) \cdot \xi_{\gamma}-\xi_{\gamma} \cdot(a, 0)\right], \\
D_{\mathcal{B}}(b) & =D((-T(b), b))=\lim _{\gamma}\left[(-T(b), b) \cdot \eta_{\gamma}-\eta_{\gamma} \cdot(-T(b), b)\right],
\end{aligned}
$$

for all $a \in \mathcal{A}$ and $b \in \mathcal{B}$. Since $\mathcal{A}$ is approximately amenable by [8, Lemma $2.2]$ there are right and left approximate identities say $\left(r_{\beta}^{\mathcal{A}}\right)$ and $\left(\ell_{\beta}^{\mathcal{A}}\right)$ respectively for $\mathcal{A}$. Similarly, let $\left(r_{\beta}^{\mathcal{B}}\right)$ and $\left(\ell_{\beta}^{\mathcal{B}}\right)$ respectively be left and right approximate identities for $\mathcal{B}$. Therefore,

$$
\begin{aligned}
(a+T(b), 0) & =\lim _{\beta}(a, b)\left(r_{\beta}^{\mathcal{A}}, 0\right)=\lim _{\beta}\left(\ell_{\beta}^{\mathcal{A}}, 0\right)(a, b), \\
(-T(b), b) & =\lim _{\beta}(a, b)\left(0, r_{\beta}^{\mathcal{B}}\right)=\lim _{\beta}\left(0, \ell_{\beta}^{\mathcal{B}}\right)(a, b),
\end{aligned}
$$

for all $a \in \mathcal{A}$ and $b \in \mathcal{B}$. Above equalities imply that there are nets $\left(\zeta_{\alpha}\right)$ and $\left(\varsigma_{\alpha}\right)$ in $X^{*}$ such that

$$
D((a, b))=D_{\mathcal{A}}(a+T(b))+D_{\mathcal{B}}(b)=\lim _{\alpha}\left[(a, b) \cdot \zeta_{\alpha}-\varsigma_{\alpha} \cdot(a, b)\right]
$$

for all $a \in \mathcal{A}$ and $b \in \mathcal{B}$. Since $D$ is a derivation we conclude that

$$
\lim _{\alpha}(a, b) \cdot\left(\zeta_{\alpha}-\varsigma_{\alpha}\right) \cdot\left(a^{\prime}, b^{\prime}\right)=0
$$

for all $a, a^{\prime} \in \mathcal{A}$ and $b, b^{\prime} \in \mathcal{B}$. Hence,

$$
D((a, b)) \cdot\left(a^{\prime}, b^{\prime}\right)=\lim _{\alpha}\left[(a, b) \cdot \varsigma_{\alpha}-\varsigma_{\alpha} \cdot(a, b)\right] \cdot\left(a^{\prime}, b^{\prime}\right)
$$

for all $a, a^{\prime} \in \mathcal{A}$ and $b, b^{\prime} \in \mathcal{B}$. Since $X$ is neo-unital it follows that

$$
D((a, b))=\text { weak }^{*}-\lim _{\alpha} \operatorname{ad}_{\varsigma_{\alpha}}((a, b))
$$

for all $a \in \mathcal{A}$ and $b \in \mathcal{B}$ as desired. 
Proposition 3.6. Let $\mathcal{A}$ and $\mathcal{B}$ be approximately amenable Banach algebras. Suppose that one of the following statements holds. Then $\mathcal{M}$ is approximately amenable.

(i) $\mathcal{A}$ is amenable.

(ii) $\mathcal{A}$ and $\mathcal{B}$ have a central approximate identity.

(iii) $\mathcal{B}$ has a bounded approximate identity.

Proof. (i). Since $\mathcal{A}$ is amenable and $\mathcal{M} / \mathcal{A} \cong \mathcal{B}$ is approximately amenable, the result follows from $[8$, Corollary 2.1] together with the fact that $\mathcal{A}$ is a closed two-sided ideal in $\mathcal{M}$.

(ii). It follows from [20, Proposition 3.4] and Theorem 2.3 that $\mathcal{M}$ is approximately biprojective in the sense of [20]. Moreover, in this case $\mathcal{M}$ has a central approximate identity. This is because of, if nets $\left(a_{\alpha}\right)$ and $\left(b_{\beta}\right)$ are central approximate identities for $\mathcal{A}$ and $\mathcal{B}$, respectively. Then the net $\left(\left(a_{\alpha}-T\left(b_{\beta}\right), b_{\beta}\right)\right)$ is a central approximate identity for $\mathcal{M}$. Indeed, for each $(a, b) \in \mathcal{M}$ we have

$$
\left(a_{\alpha}-T\left(b_{\beta}\right), b_{\beta}\right)(a, b)=\left(a_{\alpha}[a+T(b)]-T\left(b_{\beta} b\right), b_{\beta} b\right) .
$$

Hence [20, Corollary 3.7] implies that $\mathcal{M}$ is approximately amenable.

(iii). Let $X$ be a Banach $\mathcal{M}$-bimodule, and let $D: \mathcal{M} \rightarrow X^{*}$ be a continuous derivation. Suppose that $\left(b_{\alpha}\right)$ is a bounded approximate identity for $\mathcal{B}$. Without loss of generality we assume that there are $E \in \mathcal{B}^{* *}, F \in \mathcal{A}^{* *}$ and $\xi, \eta \in X^{* * *}$ such that

$$
b_{\alpha} \stackrel{w^{*}}{\longrightarrow} E, \quad T\left(b_{\alpha}\right) \stackrel{w^{*}}{\longrightarrow} F, \quad D\left(b_{\alpha}\right) \stackrel{w^{*}}{\longrightarrow} \xi, \quad D\left(T\left(b_{\alpha}\right)\right) \stackrel{w^{*}}{\longrightarrow} \eta .
$$

It is known by [6] that $X^{* * *}$ is a Banach $\mathcal{A}^{* *} \times_{T^{* *}} \mathcal{B}^{* *}$-bimodule. We can extend $T$ to a homomorphism from $\mathcal{B}$ into $\mathcal{A}^{\sharp}$, the unitization of $\mathcal{A}$, denoted still by $T$. Moreover, it is easy to show that $X^{* * *}$ is an $\mathcal{A}^{\sharp} \times_{T} \mathcal{B}$-bimodule with the module actions

$$
e_{\mathcal{A}} \cdot G=G+F \cdot G-E \cdot G, \quad G \cdot e_{\mathcal{A}}=G+G \cdot F-G \cdot E
$$

for all $G \in X^{* * *}$, where $e_{\mathcal{A}}$ is the identity element of $\mathcal{A}^{\sharp}$. We can consider $D$ as a derivation from $\mathcal{M}$ into $X^{* * *}$ and extend it to a derivation $\widetilde{D}$ from $\mathcal{A}^{\sharp} \times_{T} \mathcal{B}$ into $X^{* * *}$ defined by $\widetilde{D}\left(e_{\mathcal{A}}\right)=\eta-\xi$. For example, for each $a \in \mathcal{A}$ we have

$$
\begin{aligned}
a \cdot \widetilde{D}\left(e_{\mathcal{A}}\right)+\widetilde{D}(a) \cdot e_{\mathcal{A}} & =a \cdot \eta-a \cdot \xi+D(a)+D(a) \cdot F-D(a) \cdot E \\
& =D(a)+\text { weak }^{*}-\lim _{\alpha} D\left(\left(a T\left(b_{\alpha}\right), 0\right)-(a, 0)\left(0, b_{\alpha}\right)\right) \\
& =D(a)=\widetilde{D}\left(a e_{\mathcal{A}}\right) .
\end{aligned}
$$

Since both $\mathcal{A}^{\sharp}$ and $\mathcal{B}$ are pseudo-amenable by [9, Theorem 3.1 and Proposition 3.2], $\mathcal{A}^{\sharp} \times_{T} \mathcal{B}$ is pseudo-amenable by Proposition 3.3 and consequently again by [9, Proposition 3.2] it is approximately amenable. Thus, there is a net $\left(G_{\gamma}\right)$ in $X^{* * *}$ such that

$$
D((a, b))=\lim _{\gamma} \operatorname{ad}_{G_{\gamma}}((a, b))
$$

for all $(a, b) \in \mathcal{M}$. Applying Goldstine's theorem we can obtain $\left(G_{\gamma}\right)$ in $X^{*}$, and replace norm convergence in above equation by weak convergence. This implies, via Mazur's theorem, that $\mathcal{M}$ is approximately amenable. 
It is known that every approximately amenable Banach algebra have right and left approximate identities. However, it has been an open question whether approximately amenable Banach algebras must also have two-sided approximate identities. In the following result we show that if the $T$-Lau product $\mathcal{A} \times_{T} \mathcal{A}$ of Banach algebra $\mathcal{A}$ is approximately amenable, then $\mathcal{A}$ has a two-sided approximate identity.

Proposition 3.7. Let $\mathcal{A}$ be a Banach algebra and let $T: \mathcal{A} \rightarrow \mathcal{A}$ be a continuous homomorphism with $\|T\| \leq 1$. Then $\mathcal{A}$ has a two-sided approximate identity if $\mathcal{A} \times_{T} \mathcal{A}$ is approximately amenable.

Proof. First note that $X=\mathcal{A}$ is an $\mathcal{A} \times_{T} \mathcal{A}$-bimodule by the following module actions.

$$
(a, b) \cdot x=[a+T(b)] x \quad \text { and } \quad x \cdot(a, b)=x b
$$

for all $a, b \in \mathcal{A}$ and $x \in X$. Now, consider the linear map $D: \mathcal{A} \times_{T} \mathcal{A} \rightarrow X$ defined by $D((a, b))=a+T(b)-b$. It is not hard to check that $D$ is a continuous derivation. Applying [9, Theorem 3.1(iii)], we obtain that there is a net $\left(x_{\alpha}\right)$ in $X$ such that $D((a, b))=\lim _{\alpha} \operatorname{ad}_{x_{\alpha}}((a, b))$. It follows that

$a-b=D((a-T(b), b))=\lim _{\alpha}\left[(a-T(b), b) \cdot x_{\alpha}-x_{\alpha} \cdot(a-T(b), b)\right]=\lim _{\alpha}\left[a x_{\alpha}-x_{\alpha} b\right]$

for all $a, b \in \mathcal{A}$. Trivially $\left(x_{\alpha}\right)$ is a two-sided approximate identity for $\mathcal{A}$.

Acknowledgement. This research for the first author was in part supported by a grant from IPM (No. 92470046).

\section{REFERENCES}

1. W.G. Bade and P.C. Curtis, Homomorphisms of commutative Banach algebras, Amer. J. Math. 82 (1960), 589-608.

2. W.G. Bade and P.C. Curtis, The Wedderburn decomposition of commutative Banach algebras, Amer. J. Math. 82 (1960), 851-866.

3. W.G. Bade, P.C. Curtis and A.M. Sinclair, Raising bounded groups and splitting of radical extensions of commutative Banach algebras, Studia Math. 141 (2000), 85-98.

4. W.G. Bade and H.G. Dales, The Wedderburn decomposition of some commutative Banach algebras, J. Funct. Anal. 107 (1992), 105-121.

5. S.J. Bhatt and P.A. Dabhi, Arens regularity and amenability of Lau product of Banach algebras defined by a Banach algebra morphism, Bull. Austral. Math. Soc. 87 (2013), 195206.

6. H.G. Dales, F. Ghahramani and N. Grønbæk, Derivations into iterated duals of Banach algebras, Studia Math. 128 (1998), 19-53.

7. C. Feldman, The Wedderburn principal theorem in Banach algebras, Proc. Amer. Math. Soc. 2 (1951), 771-777.

8. F. Ghahramani and R.J. Loy, Generalized notions of amenability, J. Funct. Anal. 208 (2004), 229-260.

9. F. Ghahramani and Y. Zhang, Pseudo-amenable and pseudo-contractible Banach algebras, Math. Proc. Cambridge Philos. Soc. 142 (2007), 111-123.

10. F. Gourdeau, Amenability and the second dual of a Banach algebras, Studia Math. 125 (1997), 75-81.

11. A.Ya. Helemskii, The Homology of Banach and Topological Algebras, Kluwer Academic Publishers, Dordrecht, 1989. 
12. H. Javanshiri and M. Nemati, On a certain product of Banach algebras and some of its properties, Proc. Rom. Acad. Ser. A Math. Phys. Tech. Sci. Inf. Sci. 15 (2014), 219-227.

13. B.E. Johnson, The Wedderburn decomposition of Banach algebras with finite dimensional radical, Amer. J. Math. 90 (1968), 866-876.

14. B.E. Johnson, Cohomology in Banach Algebras, Memoirs of the American Mathematical Society, No. 127. American Mathematical Society, Providence, R.I., 1972.

15. A.R. Khoddami and H.R. Ebrahimi Vishki, Biflatness and biprojectivity of Lau product of Banach algebras, Bull. Iranian Math. Soc. 39 (2013), 559-568.

16. A.T.-M. Lau, Analysis on a class of Banach algebras with applications to harmonic analysis on locally compact groups and semigroups, Fund. Math. 118 (1983), 161-175.

17. M.S. Monfared, On certain products of Banach algebras with applications to harmonic analysis, Studia Math. 178 (2007), 277-294.

18. M.S. Monfared, Extensions and isomorphisms for the generalized Fourier algebras of a locally compact group, J. Funct. Anal. 198 (2003), 413-444.

19. A.Yu. Pirkovskii, Approximate characterizations of projectivity and injectivity for Banach modules, Math. Proc. Cambridge Philos. Soc. 143 (2007), 375-385.

20. H. Pourmahmood-Aghababa, Approximately biprojective Banach algebras and nilpotent ideals, Bull. Austral. Math. Soc. 87 (2013), 158-173.

21. Y. Zhang, Nilpotent ideals in a class of Banach algebras, Proc. Amer. Math. Soc. 127 (1999), 2337-3242.

22. Y. Zhang, Weak amenability of module extensions of Banach algebras, Trans. Amer. Math. Soc. 354 (2002), 4131-4151.

${ }^{1}$ Department of Mathematical Sciences, Isfahan Uinversity of Technology, ISFAHAN 84156-83111, IRAN;

School of Mathematics, Institute for Research in Fundamental Sciences (IPM), P.O. BOX: 19395-5746, TEHRAN, IRAN.

E-mail address: m.nemati@cc.iut.ac.ir

${ }^{2}$ Department of Mathematics, Yazd University, P.O. Box: 89195-741, Yazd, IRAN.

E-mail address: h.javanshiri@yazd.ac.ir 